\title{
Painting with Drops, Jets, and Sheets
}

\section{Citation}

Herczynski, Andrzej, Claude Cernuschi, and L. Mahadevan. 2011. Painting with drops, jets, and sheets. Physics Today 64(6): 31-36.

\section{Published Version}

doi:10.1063/1.3603916

\section{Permanent link}

http://nrs.harvard.edu/urn-3:HUL.InstRepos:5024902

\section{Terms of Use}

This article was downloaded from Harvard University's DASH repository, and is made available under the terms and conditions applicable to Other Posted Material, as set forth at http:// nrs.harvard.edu/urn-3:HUL.InstRepos:dash.current.terms-of-use\#LAA

\section{Share Your Story}

The Harvard community has made this article openly available.

Please share how this access benefits you. Submit a story.

\section{Accessibility}




\title{
Painting with drops, jets, and sheets
}

\author{
Andrzej Herczyński, Claude Cernuschi, and L. Mahadevan
}

\section{A fluid dynamics analysis of Jackson Pollock's technique opens his and other artists' work to quantitative exploration.}

Andrzej Herczyński (andrzej@bc.edu) is a research associate professor of physics and Claude Cernuschi (cernusch@bc.edu) is a professor of art history, both at Boston College in Boston, Massachusetts. L. Mahadevan (Im@seas.harvard.edu) is a professor of applied mathematics, biology, and physics at Harvard University in Cambridge, Massachusetts.

Whenever we ascribe artistic qualities to nature or natural qualities to art, we overlook crucial distinctions. It is one thing to contemplate waves crashing on the shore, candle flames flickering, or swirling whitewater rapids; quite another to transform, transcribe, or depict those scenes in a work of art; and still another to let the physical phenomena per se-waves, instability, turbulence-contribute to the making of an art object. Whether representational or abstract, all art is, in essence, artifice. And while the physical properties of materials undeniably constrain what artists can accomplish, the creative process must transcend physics or else cease to be creative. The prerogative of artists is to shape their medium-be it liquid paint, colored sand, or molten bronze-according to an aesthetic vision, to intervene rather than yield to how materials would naturally behave. As Voltaire remarked, "le secret des arts est de corriger la nature" (the secret of the arts is to correct nature). ${ }^{1}$

All the same, many artists willfully transgress the boundary between art and physics. Leonardo da Vinci's drawings of water flows and flying machines, for example, reflect such precision and methodological rigor that even present-day engineers, anatomists, and botanists consider him one of their own. The gears and pulleys in Giovanni Battista Piranesi's Carceri engravings ${ }^{2}$ may be situated in fantastical settings but appear mechanically operational. Alexander Calder's mobiles and, more recently, Richard Serra's sculptures, such as Prop, in which objects lean on each other, depend on precarious mechanical balance for their very stability and make a virtue of basic physics. Robert Morris and Eva Hesse, by using flexible materials in their hanging sculptures, offer solutions to complex problems in geometry and elasticity. Robert Smithson's glue and asphalt pourings and Lynda Benglis's poured latex and polyurethane foam sculptures owe their shapes to the flow and solidification of complex liquids.

Figure 1. Jackson Pollock (1912-56), at work in 1949, photographed by Martha Holmes. Pollock appears to be using a relatively viscous paint that forms a continuous jet of fluid he controls by moving a trowel up, down, or across the canvas. (Photograph (c) Time Inc, Getty Images.)
An innovator who tested aesthetic boundaries in a manner particularly intriguing to physicists and who influenced a generation of artists-Morris, Smithson, and Benglis among them - was the American abstract expressionist Jackson Pollock. He developed a new painting technique by pouring and occasionally dripping liquid pigment onto a canvas stretched horizontally on the floor, as pictured in figure 1. In so doing, he creatively ceded some of the responsibility for the appearance of his work to natural phenomena, inviting fluid dynamics to coauthor his pieces. Long recognized as an important achievement by art historians, ${ }^{3}$ Pollock's highly original and influential contribution has recently received closer attention from physicists interested in the scaling

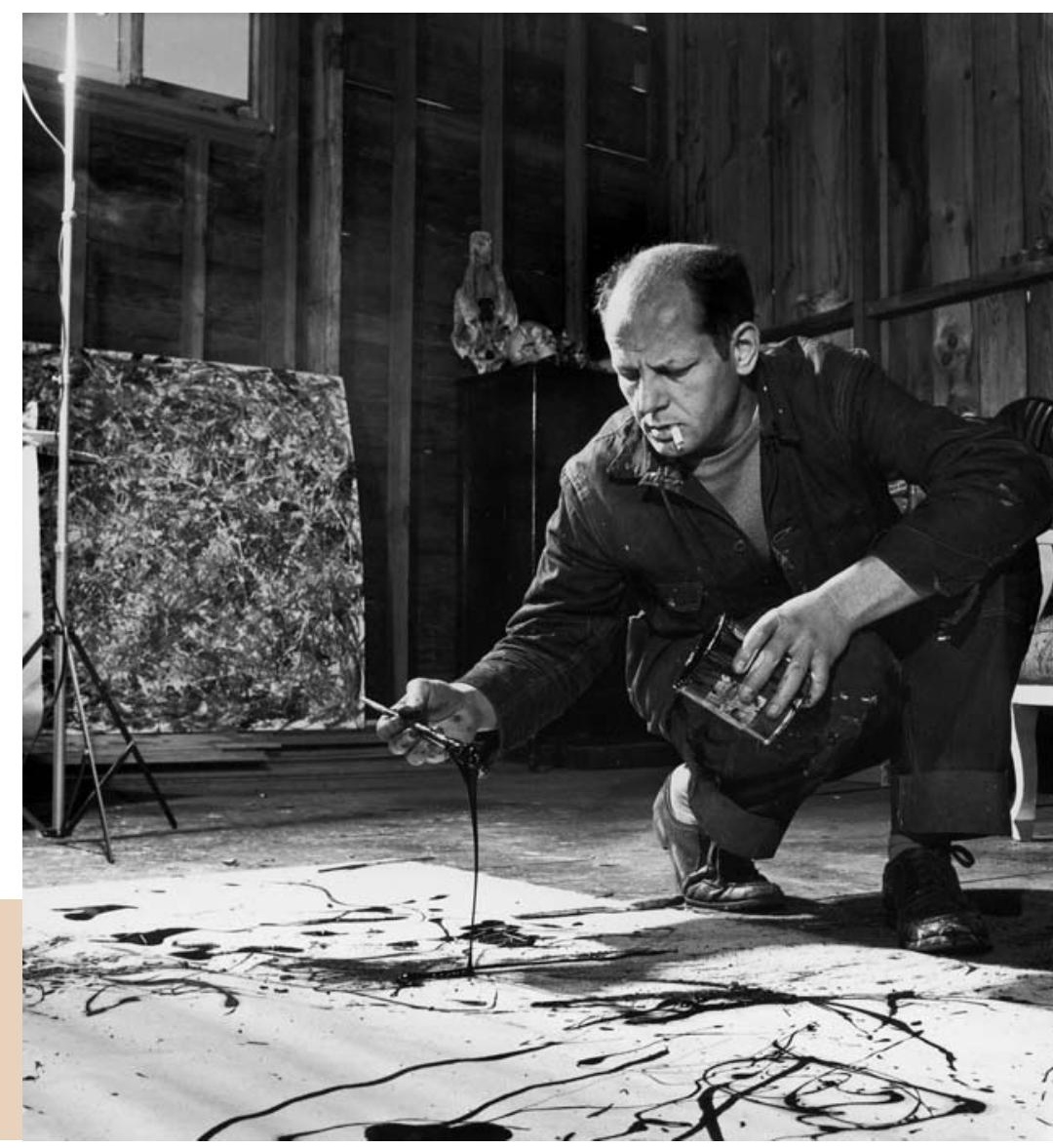




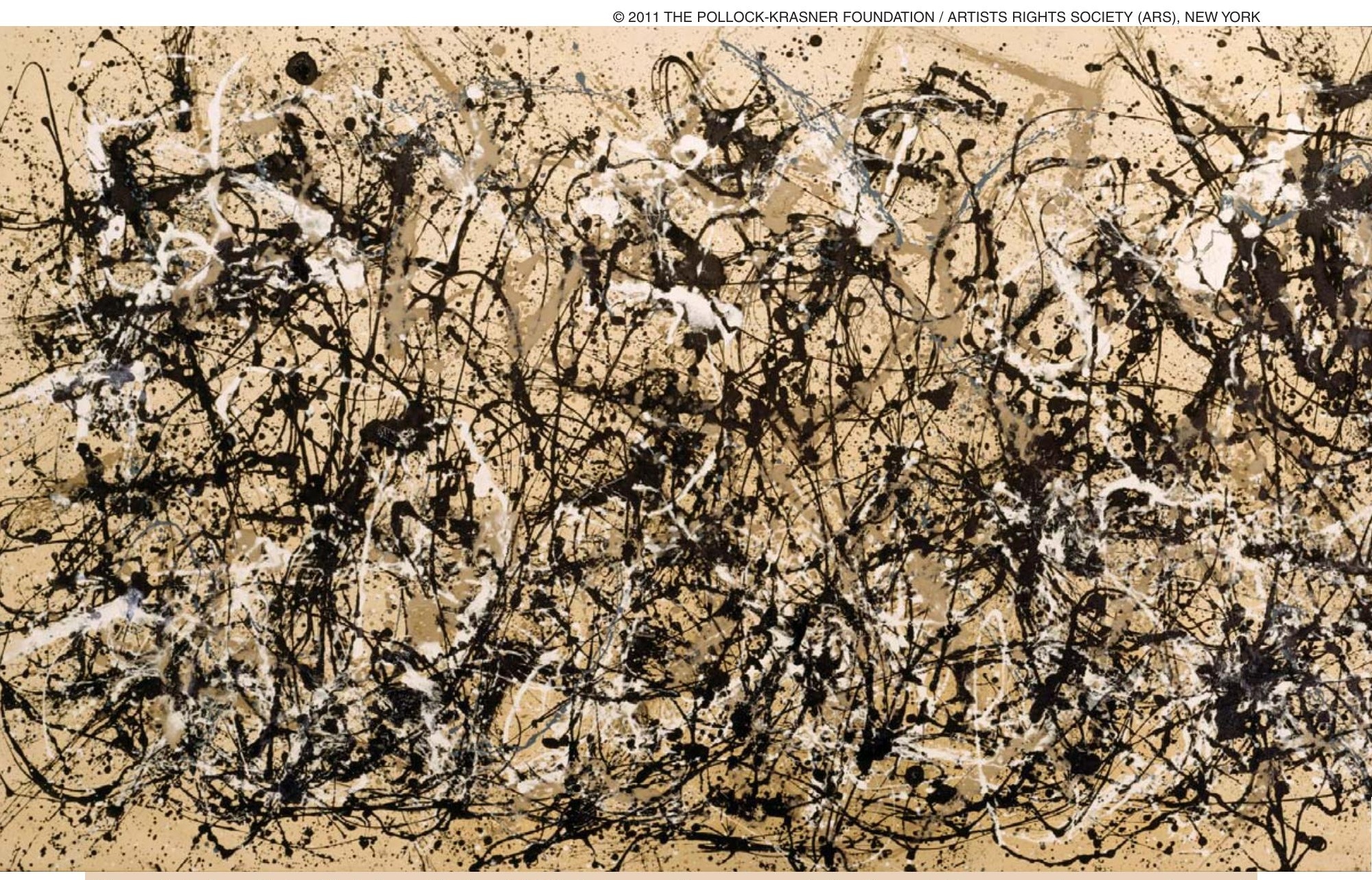

Figure 2. Jackson Pollock's Autumn Rhythm (1950) displays continuous, meandering lines-jet traces-and numerous drop and stain marks. The original artwork $(267 \times 526 \mathrm{~cm})$ hangs in the Metropolitan Museum of Art in New York.

properties of his tangled webs ${ }^{4}$ and in the physical constraints of the pouring technique.

Although the artist co-opted gravitationally driven flows to achieve his aims, the fluid dynamical aspects and implications of his process have remained largely unexplored. In this article, we examine the mechanics of Pollock's technique, including the physics of lifting and dispensing paint, and the fundamental role of instabilities in a free viscous jetthe thin, continuous thread of paint streaming from a brush or trowel.

\section{Painting with drops}

Drip painting, the label often associated with Pollock's work, suggests that he primarily relied on drops, or discrete dribbles of liquid pigment, to create his abstractions. In the popular imagination, dripping so defines the artist's modus operandi that the pejorative moniker "Jack the Dripper," coined by Time magazine in 1956, has enjoyed remarkable longevity. But while separate spots do appear in his paintings, the predominant effect of his abstract work, including such best-known pieces as the 1950 Autumn Rhythm, shown in figure 2, is that of a web of sinuous and undulating curves created by continuous filaments of paint. ${ }^{6}$

Paintings in which drop splashes dominate the composition are rare in Pollock's production, although the artist could readily have achieved that effect. The majority of the discrete marks, moreover, were most likely an accidental consequence of his painting technique, not the result of planning. It can therefore be argued that Pollock sought to have flowing, continuous lines command the spectator's primary attention and that his abstractions are more accurately described as stream paintings.

That distinction is not merely a matter of semantics. If the drop marks were more prevalent-either more prominent or simply more numerous - the visual impact would have been dramatically different, closer to the work of Sam Francis, an American artist influenced by abstract expressionism. In many of his canvases, even those not purely abstract, discrete drop splashes or sprayed dots dominate the field.

Another common assumption is that Pollock simply poured paint directly from a can or other container. Though perhaps he occasionally did so, he usually dispensed pigment in a more refined way, which provided greater control over the flow rate and produced a steadier stream for making fine lines. He dipped a stick or trowel into a can containing pigment, rapidly lifted his implement with paint adhering to it, and then let a stream run down onto the canvas below.

As the paint ran out and the flow rate decreased, the jet naturally broke into droplets. It is safe to conjecture that numerous marks were created that way-the appearance of drops perhaps reminded Pollock it was time to reload the trowel. But drops can be produced in another way: When a 
more than a meter above the canvas. The films and numerous photographs of Pollock show him comfortably manipulating the flow (see figure 1).

In choosing his particular method of dispensing pigment, Pollock gained the freedom to paint in three dimensionseven if the resulting work remained conventionally two-dimensional. He could vary the width of his traces by changing the lateral speed, the flow rate, or the height of the jet. Pollock could thus suggest sideways acceleration without actually changing the lateral speed of his implement; the art historical implications of such sleight of hand have been recently discussed. ${ }^{9}$

One intriguing, if subtle, consequence of introducing liquid-jet dynamics into the creative process is the appearance of fluid instability. As recently noted, ${ }^{9}$ a number of Pollock's works on paper display fine oscillatory lines of very short wavelength (a few millimeters). Those traces almost certainly resulted from a coiling and folding instability of the viscous jet, superposed on a linear translation of trowel. Indeed, the coiling instability is particularly conspicuous in some of the red lines in Untitled 1948-49, shown in figure 5. Although Pollock's exact motivations are unknown, the effect likely resulted from a combination of pure chance and guided inquiry. Perhaps seeing that a stream of red enamel paint autonomously produced the fine but distinct undulations, he set out to playfully explore them. Remarkably, the inquisitive exploration preceded the first papers on the physics of coiling. ${ }^{10,11}$

A quantitative explanation for the folding and coiling instabilities in falling viscous streams and jets was published more recently; a scaling law for the inertial regime, in which viscous forces and inertia are balanced in the coiling tail, was first derived and experimentally verified just over a decade ago. ${ }^{12}$ Two other regimes of coiling can be distinguished: a viscous regime, in which gravity and inertia are negligible in the coil and the net viscous force on any liquid element is zero-a situation akin to the folding of a toothpaste stream onto a toothbrush - and a gravitational regime, in which viscous forces are balanced by gravity. ${ }^{13}$

The coiling effect in Untitled 1948-49 corresponds to the scaling law for the inertial regime, which gives the frequency $\omega$ of the coiling tail as

$$
\omega \sim v^{-1 / 3} r^{-10 / 3} Q^{4 / 3},
$$

where $r$ is the radius of the jet's tail (see the box on page 35). Expression 4 is valid when two conditions are satisfied. First, the height of the trowel above the floor must be large relative to the jet radius, as otherwise the coil radius $R$ is proportional to the height $H$ of the jet. The maximum radius of oscillations in the red enamel traces shown in figure 5 is less than $0.3 \mathrm{~cm}$, so $H \gg R \geq r$. Second, gravitational forces must be small compared to the inertia of the coiling tail, so that $g \ll \omega^{2} R$, which can be reformulated as $Q^{2} \gg g R r^{2}$. Assuming a typical flow rate, $Q=1 \mathrm{~cm}^{3} / \mathrm{s}$, coil radius $R \sim 0.3 \mathrm{~cm}$, and half line width $r \sim 0.05 \mathrm{~cm}$, we see that the second condition is also easily satisfied.

\section{Reading jet traces}

A work on paper, Pollock's Untitled 1948-49 is executed not b

Figure 4. (a) A cylindrical rod of radius $r_{0}$ acts as a trowel, entraining viscous liquid with a thickness $h$ as the rod is pulled upward with speed $u_{0}$ out of a paint can. (b) When the liquid-coated rod emerges from the can, a gravitationally driven jet of viscous liquid flows from the end of the rod at a rate $Q$.

only in red enamel paint but also in black ink. The black marks display no coiling instability whatsoever, presumably because they were created with diluted ink of low viscosity, comparable to that of water. Hence, whereas the red traces are glossy and slightly protrude from the canvas, those in black are matte and planar, the ink having partially seeped into the paper.

To explore the implications of equation 4 for the coiling effects manifest in the red skeins, it is convenient to eliminate $\omega$-which, after all, cannot be measured post factum-and find scaling laws for two principal observable parameters: the line half-width $r$ and the coil radius $R$. Because the volume of the paint is conserved in its transport from the implement to surface, $Q \sim u_{0} r_{0}^{2}=u r^{2}$, where $u_{0}$ and $u$ are, respectively, the speed of the jet at the origin and its speed at the point of contact with the canvas, while $r_{0}$ and $r$ are the respective jet radii at those locations. By balancing the gravitational force and the viscous resistance to stretching, and using the results in equation 4 after setting $Q \sim u r^{2} \sim \omega R r^{2}$, we obtain

$$
r \sim \frac{1}{H}\left(\frac{v Q}{g}\right)^{1 / 2} \text { and } R \sim v\left(\frac{Q}{H^{4} g^{2}}\right)^{1 / 3}
$$

Those scalings can be used to estimate the range of flow rates that Pollock produced when painting Untitled 1948-49. From the enlargement of the work, the coil radius can be seen to vary by roughly a factor of 4 , between 0.5 and $2 \mathrm{~mm}$. Based on the second scaling in equation 5, the flow rate $Q$ thus varied by nearly two orders of magnitude-somewhere in the range of $0.1-10 \mathrm{~cm}^{3} / \mathrm{s}$ - assuming a fixed height. Accordingly, using the first of the scalings in equation 5, the thickness of the oscillating lines should have varied by about one order of magnitude or more; and indeed, the observed range is approximately $0.1-1 \mathrm{~mm}$. Further, taking $r=0.5 \mathrm{~mm}$ as a typical value for this painting, we estimate $Q / H^{2} \approx 2.5 \times 10^{-3} \mathrm{~cm} / \mathrm{s}$, so that if the artist kept his trowel at about $30 \mathrm{~cm}$ above the painting (as suggested by figure 1), the flow rate in the jet might have been about $2 \mathrm{~cm}^{3} / \mathrm{s}$, a reasonable value.

A close look at figure 5 reveals several instances in which the coiling oscillations become less distinct and eventually 


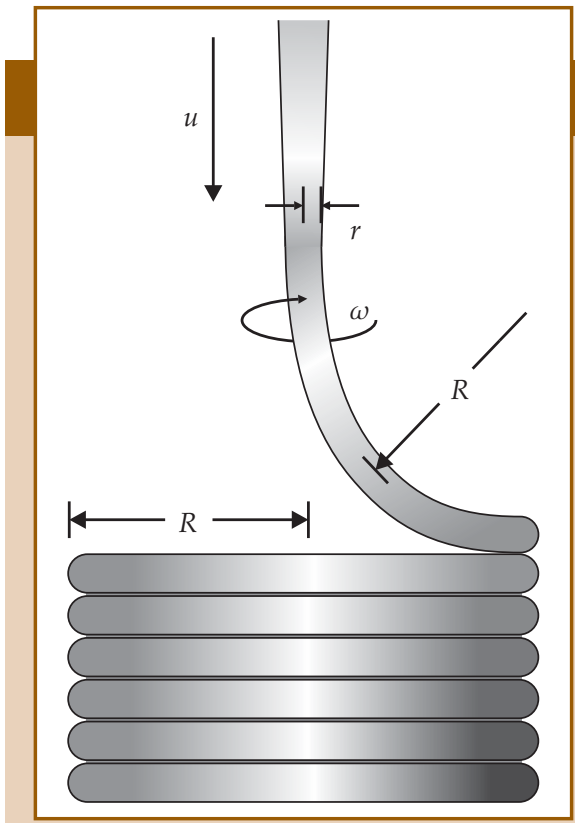

Dynamics of coiling: A scaling argument

When viscous paint is poured onto a surface from a relatively large height $H$, the instability of the fluid stream causes it to form a helical coil whose radius and frequency are determined by the balance of viscous and inertial torques. To understand that balance quantitatively, we start with an equation for the conservation of material: $Q \sim \omega R r^{2}$, which relates the flow rate $Q$ to the radius $R$ of the coil, its rate of rotation $\omega$, and the radius $r$ of the paint stream.

The expression for the viscous torque, $\mu u r^{4} / R^{2}$, characterizes the stream's resistance to bending, which is proportional to the viscosity $\mu$ of the paint, the fourth power of its radius, and the rate of change of its curvature $u / R^{2}$, where $u$ is the paint's axial velocity. This equation follows from dimensional analysis, or the Stokes-Rayleigh analogy relating the equations of equilibrium for an elastic solid and the equations of motion for the creeping flow of a viscous liquid, as described in Lord Rayleigh's The Theory of Sound..$^{15}$ (The kinematic viscosity $v$ can be expressed in terms of the fluid's density $\rho$ as $v=\mu / \rho$.)

The expression for the inertial torque, $\left(\rho \omega^{2} R\right) \cdot r^{2} R \cdot R$, characterizes the Coriolis and centripetal torques; the term in parentheses is the inertial force per unit volume, which acts on a volume of liquid $r^{2} R$. Equating the viscous and inertial torques and using the relation $u=\omega R$ leads to the scaling for the coiling frequency: $:^{12} \omega \sim Q^{4 / 3} r^{-10 / 3} v^{-1 / 3}$.

blur together as the line thickens. The scaling relations in equation 5 offer an explanation. Note that for a given paint (and thus $v$ ) and at a fixed jet height $H$, the width $2 r$ of the line grows more quickly with $Q$ than does the amplitude $R$ of the oscillations: $r / R$ is proportional to $Q^{1 / 6}$. If the flow rate tripled at some instant - for example, due to an accumulation of paint on the trowel-the ratio $r / R$ would rise by $20 \%$ and render any oscillations in the trace hard, if not impossible, to observe. (In any case, the sudden influx of paint would likely disturb the coiling instability.)

We now turn our attention to the shape of the undulating lines in Pollock's paintings. When coiling (the circular motion of the tail of the paint jet) is superposed with transverse motion (the sweeping lateral movement of Pollock's arm), the resulting trace depends on a dimensionless quantity known as the Strouhal number $S t=U / \omega R$, where $\omega$ is the angular speed of coiling as given by equation 4 and $U$ is the transverse speed of the paint.

When $S t=0$, the trace coils into a circle. But as $S t$ increases, the trace becomes a series of overlapping loops of ever decreasing overlap until $S t=1$, when the trace becomes a curve with evenly spaced cusps. When $S t$ increases beyond unity, the trace is an oscillating quasi-sinusoidal line that becomes straighter as the transverse velocity increases.

A viscous thread of paint that's held stationary but that falls on a moving surface-the inverse of the scenario executed by Pollock - would engender a richer variety of autonomous instability effects, including loops that alternate between the two sides of an undulating line and other twofrequency traces, such as a sequence of figure eights. The physics of that meandering instability, or fluid dynamical "stitching," has been recently explored using experimental, semianalytical, and numerical methods, ${ }^{14}$ for a video of a viscous thread falling on a moving belt, see the online version of this article.

Whether a jet's origin is moving above a stationary surface or is stationary above a moving surface may seem to be, at first glance, a simple choice of coordinate system for describing the flow. The two scenarios, however, are not equivalent from the perspective of understanding how the instabilities evolve in space and time-especially in an inertial setting because of the difference in how the boundaries are forced. The different scenarios thus lead to different strate- gies for controlling the patterns that emerge, the natural goal of a painter, even if those patterns are similar.

All three of the possible kinds of traces that can form on a stationary surface-loops, cusps, and sinusoids-are clearly visible in the inset detail of Untitled 1948-49. Indeed, it is possible to decipher the variations in transverse speed $U$ of Pollock's arm from the changing form of his traces. (For many of the lines, variations in $r$ are imperceptible over 10to $20-\mathrm{cm}$ stretches, and one can suppose that $Q$ was nearly constant in those instances.) In particular, one can deduce that Pollock's arm was slowing down while turning, as one would expect, and that he purposefully varied the conditions in order to elicit coiling instability in his viscous jetsdelighting, one might imagine, in deploying the effect repeatedly in that work.

The ease with which coiling instabilities are obtained raises the question as to why they appear in just a few of Pollock's poured abstractions. Barring direct knowledge of the artist's intentions, one can only speculate. On the one hand, Pollock's jets of paint may have coiled, but the instability left no trace in the finished work-because of the irregularity of the surface (from, for example, the weave of the canvas), the pigment's absorption into the substrate, or subsequent layers of paint covering the initial marks. On the other hand, inasmuch as Pollock might have taken pleasure in amending $\mathrm{Un}_{n}$ titled 1948-49 with coiling, he might also have concluded that creating those oscillations ceded too much of his control to natural phenomena.

\section{Painting with sheets}

If drops and jets generate a rich variety of artistic effects, can the same be said of gravitationally driven thin films of paint? Although it is easy enough to envision such a scenario, we have yet to find any examples of art unambiguously fashioned in that way. To be sure, Smithson and Benglis created sculptures using quasi-2D laminar flows that later solidified on the ground or on wire scaffolding. But those works were not, for the most part, free-falling flows, nor could they be easily manipulated, and the possibilities opened by the artists' approach, at least from a physics perspective, seem limited.

Pollock himself did not paint with sheets, except in those rare instances when a narrow film of pigment would have 


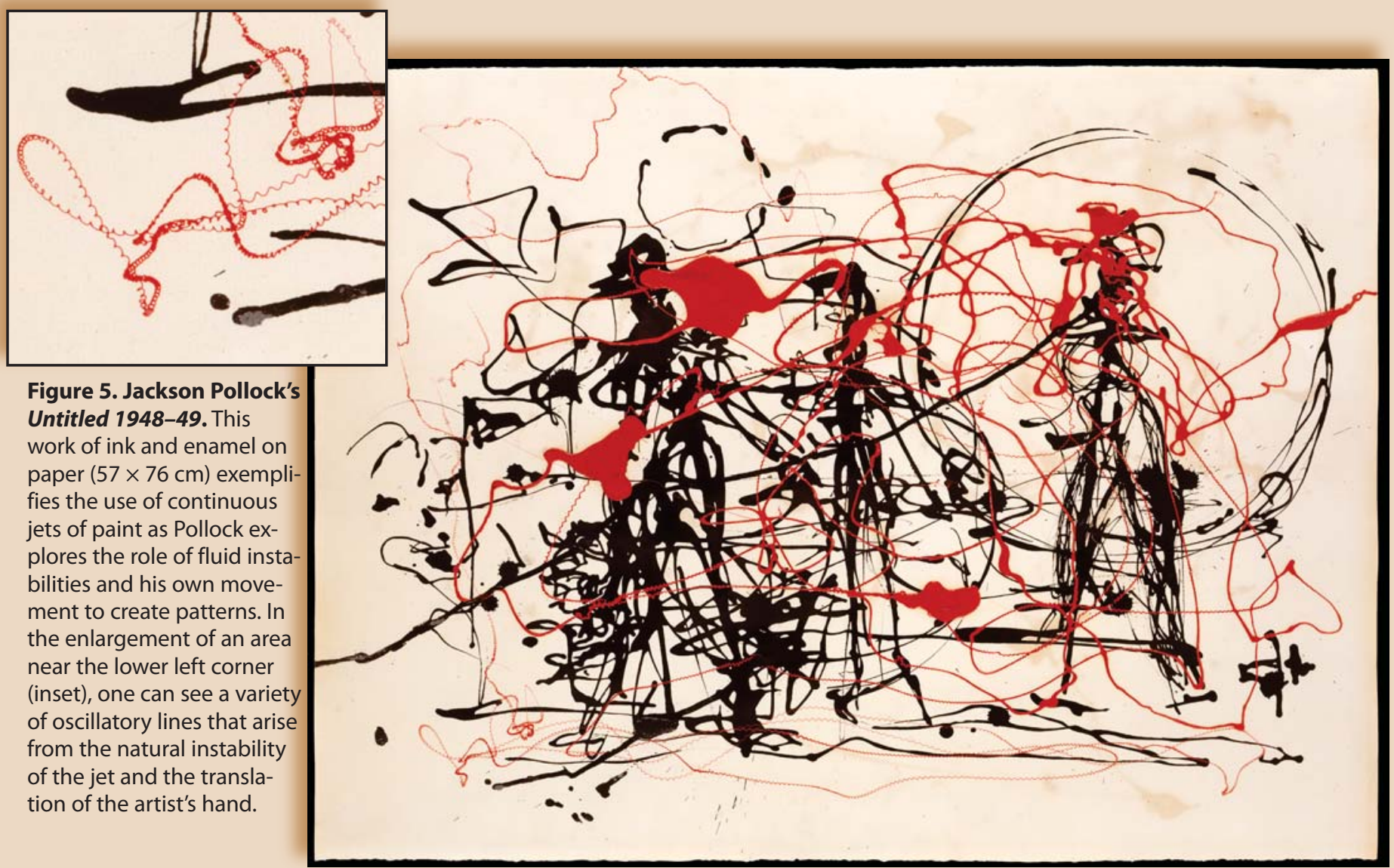

( 2011 THE POLLOCK-KRASNER FOUNDATION / ARTISTS RIGHTS SOCIETY (ARS), NEW YORK

released from his trowel tilted away from the vertical. And such instances are hard to detect in his abstractions. In any case, his particular technique was not easily adaptable to free-falling sheets. He would have required wide implements and, by extension, a number of sufficiently large paint containers. And manipulating such tools would have been cumbersome. But even if those challenges were met, the paint sheets produced would soon break into separate jets due to surface-tension instabilities. And as the paint ran out, those continuous jets would, in turn, break into cascades of discrete drops.

Perhaps a more effective way of painting with a sheet of pigment would be to let it issue from a narrow slit in an elongated container. Such a film of viscous liquid could, for sufficiently large drop height, exhibit a quasi-2D folding instability, ${ }^{13}$ but the visual effects obtainable by manipulating films of paint, and their aesthetic appeal, remain to be explored.

Whatever directions artists take, their work ultimately stands apart from nature. But to the extent that they relinquish some degree of control to natural phenomena, their art becomes open to quantitative analysis. Fluid dynamical considerations not only clarify the distinctions among various modes of gravity-assisted painting and the limitations and potential of each, they also couple the movements of the artist to the dynamics of the medium. Using the tools of physics and art history one may begin to delineate the intersection of what is aesthetically viable and what is physically possible.

\section{References}

1. Voltaire, Oeuvres Complètes de Voltaire, vol. 10, Garnier Frères, Paris (1877), p. 513.
2. See, for example, The Drawbridge, Carceri Plate VII, in L. Ficacci, Piranesi: The Complete Etchings, Taschen, Cologne, Germany (2000), p. 141

3. See C. Cernuschi, Jackson Pollock: Meaning and Significance, 1st ed., Harper/Collins, New York (1992), chap. 7.

4. R. P. Taylor, A. P. Micolich, D. Jonas, Nature 399, 422 (1999); R. P. Taylor, A. P. Micolich, D. Jonas, Leonardo 35, 203 (2002); K. JonesSmith, H. Mathur, Nature 444, E9 (2006); K. Jones-Smith, H. Mathur, L. Krauss, Phys. Rev. E 79, 046111 (2009); C. Cernuschi, A. Herczyński, D. Martin, in Pollock Matters, E. G. Landau, C. Cernuschi, eds., McMullen Museum of Art, Boston College, Chestnut Hill, MA (2007), p. 91

5. C. Cernuschi, A. Herczyński, in Pollock Matters, E. G. Landau, C. Cernuschi, eds., McMullen Museum of Art, Boston College, Chestnut Hill, MA (2007), p. 73.

6. C. Cernuschi, "Not an Illustration but the Equivalent": A Cognitive Approach to Abstract Expressionism, Fairleigh Dickinson U. Press, Madison, NJ (1997), p. 127.

7. S. D. R. Wilson, J. Fluid Mech. 190, 561 (1988).

8. H. K. Moffatt, J. Mec. 16, 651 (1977).

9. C. Cernuschi, A. Herczyński, The Art Bulletin XC, 616 (2008).

10. G. Barnes, R. Woodcock, Am. J. Phys. 26, 205 (1958).

11. G. I. Taylor, Proceedings of the 12th International Congress in Applied Mechanics, Springer, Berlin (1969), p. 382.

12. L. Mahadevan, W. S. Ryu, A. D. T. Samuel, Nature 392, 140 (1998); correction, Nature 403, 502 (2000).

13. M. Skorobogatiy, L. Mahadevan, Europhys. Lett. 52, 532 (2000); N. M. Ribe, Proc. R. Soc. London A 460, 3223 (2004).

14. S. Chiu-Webster, J. R. Lister, J. Fluid Mech. 569, 89 (2006); N. M. Ribe, J. R. Lister, S. Chiu-Webster, Phys. Fluids 18, 124105 (2006); S. W. Morris, J. H. P. Dawes, N. M. Ribe, J. R. Lister, Phys. Rev. E 77, 066218 (2008)

15. J. W. Strutt (Lord Rayleigh), The Theory of Sound, 2nd ed., Dover, New York (1945). 